\title{
Differentiation of cirrhosis from normal liver based on textural features via T1WI computer-aided diagnosis with a genetic algorithm
}

\author{
Dong-mei Guo ${ }^{2, a}$, Hui Liu ${ }^{1, b^{*}}$, Tian-shuang Qiu ${ }^{1, c}$, Xiang-bo Lin ${ }^{1, d}$ \\ ${ }^{1}$ Department of Biomedical Engineering, Dalian University of Technology, Dalian 116024, China; \\ ${ }^{2}$ Department of Radiology, Second Affiliated Hospital, Dalian Medical University, Dalian 116027, \\ China

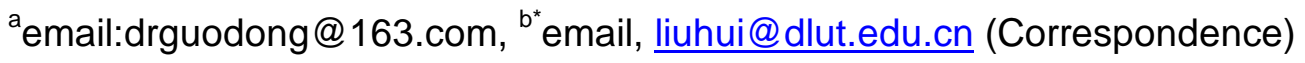 \\ cemail: giutsh@dlut.edu.cn, demail: linxbo@dlut.edu.cn
}

\begin{abstract}
Keywords: Cirrhosis; Magnetic resonance imaging; Texture feature; BP classifier; Genetic algorithm.

Abstract. A computer-aided diagnosis (CAD) system for classification of liver cirrhosis from MRI is presented. The system consists of feature extraction and selection, classification, and classifier optimization modules. In general, biomedical imaging is based on textural features, visualized via grey level co-occurrence matrices. However, these features are so numerous that it is difficult to determine which are the most effective for classification. Then feature selection was facilitated by application of a box plot. In addition to ensure the stability of the back-propagation (BP) classifier and improve its performance, a genetic algorithm (GA) was incorporated. We demonstrated that the proposed CAD system is suitable for differentiation through analysis of 170 regions of interest in T1WIs of advanced cirrhosis and normal livers. The GA improved classification performance of the BP classifier, allowing fewer iterations, less time expense, and a high accuracy rate.
\end{abstract}

\section{Introduction}

Hepatic cirrhosis is a late stage of progressive hepatic disease and its early detection and accurate staging is an important issue in practical radiology. Magnetic resonance imaging (MRI) has been a sensitive and robust imaging technique for detection cirrhosis noninvasively, but it is difficult to diagnose early stage cirrhosis by non-enhanced MRI. Early stage cirrhosis often requires confirmation with enhanced MRI by administrating contrast agents, or invasive hepatic biopsy ${ }^{[1]}$.

A computer-aided detection (CAD) system can be employed to assist the radiologist in staging hepatic diseases. CAD systems may eventually reduce the number of administrations of contrast agents and hepatic biopsies ${ }^{[2]}$. Recently, a few studies have focused on the clinical use of CAD to support and improve MRI differentiation of cirrhosis. Zhang et al. ${ }^{[3]}$ used an artificial neural network (ANN) classifier based on liver shape and textural features to differentiate cirrhotic and normal liver, resulting in a correct detection of cirrhosis of $82.00 \%$. Jiang et al. ${ }^{[4]}$ also employed an ANN classifier in which shape and textural features were integrated to differentiate normal and cirrhotic liver, with a success rate of $90.00 \%$. In our previous study ${ }^{[5]}$, we used an ANN classifier that incorporated six parameters of textural characteristics to classify normal and hepatocellular carcinoma in a rat model, and the correct classification rate was $91.67 \%$.

The impetus of the present paper was to improve the accuracy of the ANN classifier for cirrhosis diagnosis, making classification more stable and reliable, with the eventual goal of diagnosing hepatic cirrhosis in early stages. Firstly, the characteristics of 56 textural features were extracted, and feature selection conducted via a box plot. Then, we designed an ANN classifier to differentiate normal from cirrhotic liver. The ANN classifier was further optimized with a genetic algorithm $(\mathrm{GA}){ }^{[6]}$. We chose non-enhanced T1-weighted images (T1WI) for analysis, because T1WI is the most useful sequence for diagnosing hepatic cirrhosis in clinic. We chose advanced cirrhosis as the research object for the present preliminary study, because it is difficult to collect sufficient MRIs from early stage cirrhosis 
patients within a short time. This study is preparation for the next step of our research in MRI-CAD of early stage hepatic cirrhosis.

\section{Methods}

System outline. The proposed CAD system consists of three basic modules: feature extraction/selection, classification, and classifier optimization. All datasets are stored in a personal computer (Pentium Dual-Core2.50 GHz with 1.99GB RAM). MATLAB 6.5 was used to implement the ANN classifier.

Image acquisition. We choose to analyze the T1WIs of 18 patients, comprising 10 advanced cirrhosis and 8 normal liver patients. The T1WIs were obtained from Second Affiliated Hospital of Dalian Medical University. A 1.5T Signa MR scanner was used for image scanning. The parameters of the T1WI sequence were: TR $175.0 \mathrm{~ms}$; TE $4.2 \mathrm{~ms}$; matrix $320 \times 224$; slice thickness $8 \mathrm{~mm}$; and inter-slice gap $2 \mathrm{~mm}$. Image size was $256 \times 128$ pixels. A rectangular region of interest (ROI; $8.0 \mathrm{~mm}$ $\times 8.0 \mathrm{~mm}$ ) was segmented out manually by an experienced radiologist, avoiding major vascular structures in the ROI. A total of 170 free-hand ROIs ( 88 corresponding to cirrhosis, 82 to normal) were sampled and distributed into the next step CAD process.

The T1WI of normal liver tissue shows a hepatic surface that is regular, the hepatic signal is isointense, and the image texture appears clear and delicate without focal nodules. The T1WI of advanced hepatic cirrhosis shows a hepatic surface that is irregular, with a morphology that includes diffuse mild hyperintense or isointense nodules. Fibrosis appears as thin or thick bands that surround regenerative nodules (Fig. 1).

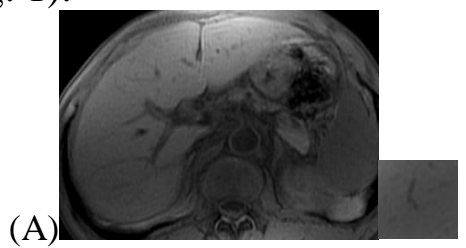

(B)

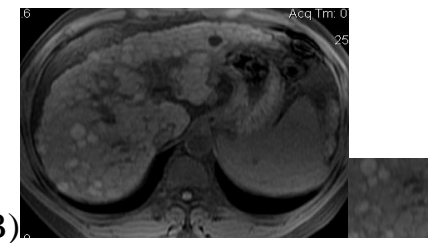

Fig.1 Non-enhanced $\mathrm{T}_{1}$ WI ROI of normal liver (A) and Cirrhosis (B)

Feature extraction and feature selection. Textural feature characteristics were extracted by grey level co-occurrence matrices ${ }^{[7]}$. Each image is a matrix of relative frequencies, $P_{i, j}(d, \theta)$, within which two neighboring pixels are separated by distance $d$ at an angle $\theta$ on the image, one with grey level $i$ and the other with grey level $j$. Fourteen textural features were derived as the following: Angular second moment, Contrast, Correlation, Variance, Inverse difference moment, Sum average, Sum variance, Sum entropy, Entropy, Difference variance, Difference entropy ,Information measures of correlation $(1,2)$, Maximal correlation coefficient.

For each ROI, each of the above 14 textural features were calculated from $\mathrm{d}=1$ and 4 directions $\theta$ equaling to $0^{\circ}, 45^{\circ}, 90^{\circ}$, and $135^{\circ}$. Therefore, for each ROI, 56 textural features were obtained. Then the feature selection was carried out by a box plot. The textural features were chosen with the goal that they can be able to differentiate clearly between cirrhotic and normal tissue without the confusion of overlap.

Classification. A back-propagation (BP) classifier was designed. The BP classifier is a conventional three-layer feed-forward neural network, consisting of one input layer, one hidden layer, and one output layer. The input layer is preferably a differentiating textural feature. In this paper, the hidden layer was designed with 8 units. The output layer classifies cirrhosis or normal. The transfer function is sigmoid. The learning step was 25 , momentum factor 0.9 , expected distortion $1 \times 10^{-6}$, and maximum training cycle 5000. Then the available dataset was randomly partitioned into a training set and a testing set. The training set consisted of 110 ROIs (cirrhosis 58, normal 52), and the testing set was the balance of 60 ROIs (cirrhosis 30, normal 30).

Optimization. The targeted function of the BP neural network is multi-apex, with different weights to initialize the network, and the network will constringe to different local minimums. At the same time, 
the GA is an effective solution to do the parallel general searching work. The GA has many applications for solving complex general optimization problems. GA-BP is a combination of the GA and BP neural network. The GA approximates a general optimization solution for the weight, and initializes the BP by the solutions above. This effectively avoids the dependence of BP on the original weight.

In this paper the differentiable textural characteristics of the liver are fed to the BP and the GA-BP classifiers to train and test, respectively.

\section{Results and Discussion}

Texture analysis. Twenty-four textural features including four directions $\left(0^{\circ}, 45^{\circ}, 90^{\circ}, 135^{\circ}\right)$ and angular second moment, contrast, correlation, inverse difference moment, sum variance, and difference variance were found able to differentiate cirrhotic from normal liver tissue after texture extraction and texture selection (Fig. 2). These features were chosen to feed the BP and the GA-BP classifiers.
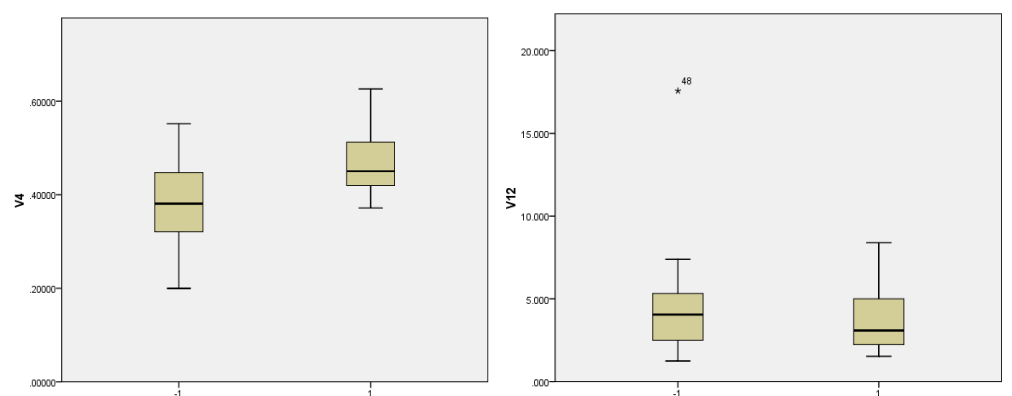

Fig.2 Box Plot of Contrast (V4) shows preferably separability texture feature. Box Plot of Difference Entropy (V12) shows confusion texture feature.

Performance of BP classifier and GA-BP classifier. The accuracy, sensitivity, and specificity of the GA-BP classifier was similar to that of the BP classifier (Table 1). It was verified that the characteristic textural features, after feature selection, could effectively translate the MRI data to differentiate cirrhosis from normal liver tissue.

Table.1 Result of performance evaluation

\begin{tabular}{cccc}
\hline Group & Sensitivity & Specificity & Accuracy \\
\hline GA-BP & 0.93 & 0.95 & 0.94 \\
BP & 0.96 & 0.93 & 0.95 \\
\hline
\end{tabular}

The average convergence time of the GA-BP classifier was $1.42 \mathrm{~ms}$ (range, 1.04 to $1.92 \mathrm{~ms}$ ), and the average convergence time of the BP classifier was $2.46 \mathrm{~ms}$ (range 1.95 to $3.25 \mathrm{~ms}$ ). Thus, the average convergence time of the GA-BP classifier is faster than that of the BP classifier. The GA-BP classifier requires fewer iterations than the BP classifier (Fig. 3 and Fig. 4)). The maximum, minimum, and average number of iterations of the GA-BP classifier was 77, 48, and 65, respectively, while those of the BP classifier was 107, 87, and 91.75. This indicates that the GA-BP classifier is more stable than the BP classifier.

Our study is limited in that only T1WIs of advanced cirrhosis were chosen; examples of early stage cirrhosis were not included. The next steps in our research will be to expand the capability of the GA-BP classifier to differentiate early stage cirrhosis on T1WI, and to interpret other MRI sequences.

\section{Conclusion}

In this study we designed and implemented a BP classifier, based on characteristic liver textural features and optimized by a GA, to discriminate patient hepatic cirrhosis from normal liver tissue. The results show that characteristic textural features after feature selection are suitable for discriminating 
patient hepatic cirrhosis from normal on T1WI, and the GA-BP classifier is more stable than the BP classifier.
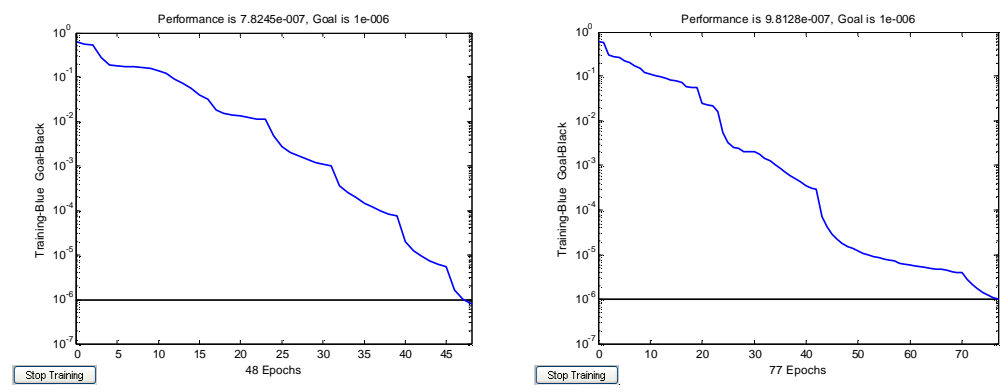

Fig. 3 Training processing of GA-BP classifier
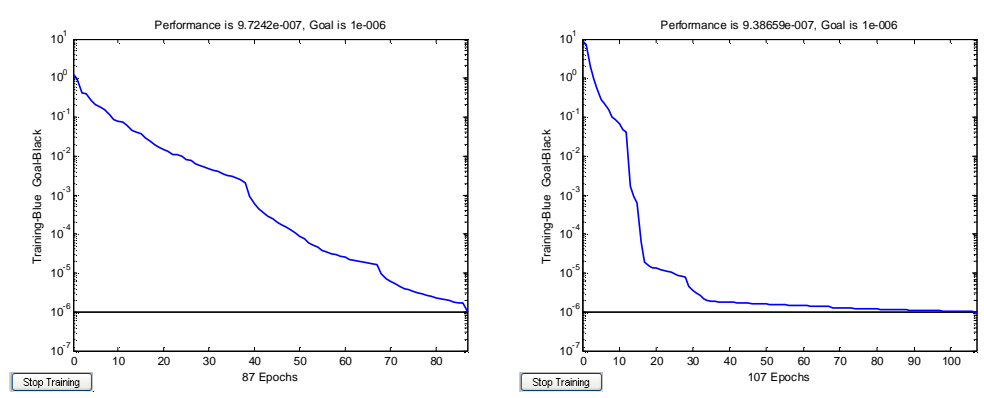

Fig. 4 Training processing of the BP NN classifier

\section{Acknowledgement}

This work is supported by" the Fundamental Research Funds for the Central Universities"

\section{References}

[1] Bonekamp S, Kamel I, Solga S, Clark J. Can imaging modalities diagnose and stage hepatic fibrosis and cirrhosis accurately. Journal of Hepatology. 2009,50:17-35.

[2] Zhang XJ, Fujita H, Qin T, Zhao JC, Kanematsu M, Hara T, et al. CAD on Liver Using CT and MRI. LNCS 2008, 4987, 367-376.

[3] Zhang XJ, Fujita H, Kanematsu M, Zhou XR, Hara T, Kato H. et al .Improving the classification of cirrhotic liver by using texture features [C].Engineering in Medicine and Biology 27th Annual International Conference. Shanghai, 2005, 867-870.

[4] Jiang HY, Zhao Y, Yang FX. Computer aided diagnosis on hepatic cirrhosis degree based on MRI[J]. Journal of Northeastern University. (Natural Science).2007,28(1):31-34.

[5] Guo DM, Qiu TS, Bian J, Kang W, Zhang L.A computer-aided diagnostic system to discriminate SPIO-enhanced magnetic resonance hepatocellular carcinoma by a neural network classifier. Computerized Medical Imaging and Graphics.2009,33: 588-592.

[6] Goldberg D, Genetic Algorithms in Search, Optimization and Machine Learning: Addison-Wesley, 989.

[7] Haralick RM, Shanmugam K, Dinstein I. Textural features for image classification [J]. IEEE Trans Syst Man Cybernet [J]. 1973; 3:610-21. 